\title{
PSMA PET-CT outperforms conventional imaging in high-risk prostate cancer
}

\section{PSMA PET-CT demonstrated 92\% accuracy compared with $65 \%$ accuracy for conventional imaging}

A new trial in men with newly

diagnosed high-risk prostate

cancer shows that prostate-specific membrane antigen (PSMA) PET-CT has superior diagnostic accuracy for detection of pelvic nodal or distant metastases to conventional imaging with $\mathrm{CT}$ and bone scanning.

PSMA PET-CT has shown great promise to improve prostate cancer imaging, particularly in patients with recurrent cancer, but evidence from multicentre, randomized trials is lacking. A team of researchers and clinicians from Australia have now addressed this lack of data in the proPSMA trial, which was recently published in The Lancet. "We designed a randomized, controlled study in order to produce the highest level of evidence and reduce bias in evaluating a head-to-head analysis of PSMA PET-CT to conventional imaging," explains Michael Hofman, lead author of the paper. "We chose accuracy as the primary end point, as providing an accurate diagnosis is the key purpose of an imaging test." Secondary end points included effects on patient management, rate of uncertain findings, value of second-line imaging with the alternative imaging modality and radiation exposure.

At ten trial sites, 300 men with histopathologically confirmed prostate cancer due to undergo radical prostatectomy or radiotherapy with curative intent received either first-line PSMA PET-CT or CT

and bone scanning following random assignment. Men in whom $<3$ unequivocal distant metastases were identified on first-line imaging underwent second-line imaging with the other imaging modality. This cut-off was chosen to avoid unnecessary procedures, as the identification of additional metastatic sites in men with widespread disease was deemed unlikely to provide patient benefit. Median patient age was 68.1 years, $27 \%$ of men had $\geq \mathrm{cT} 3$ disease, $98 \%$ of men had disease classified as ISUP grade group $\geq 3$ and characteristics were overall similar in the two imaging groups.

PSMA PET-CT demonstrated $92 \%$ accuracy compared with $65 \%$ accuracy for conventional imaging (area under the curve (AUC) difference 27\%, 95\% CI 23-31\%; $P<0.0001)$. Conventional imaging showed lower sensitivity and specificity than PSMA PET-CT (38\% versus $85 \%$ and $91 \%$ versus $98 \%$, respectively). In patient subgroup analyses, PSMA PET-CT was superior to conventional imaging in men with pelvic nodal metastases (91\% versus 59\%) and distant metastases (95\% versus $74 \%$ ). Uncertain results in the identification of any metastatic disease were more common for conventional imaging (23\% of men) than for PSMA PET-CT (7\% of men).

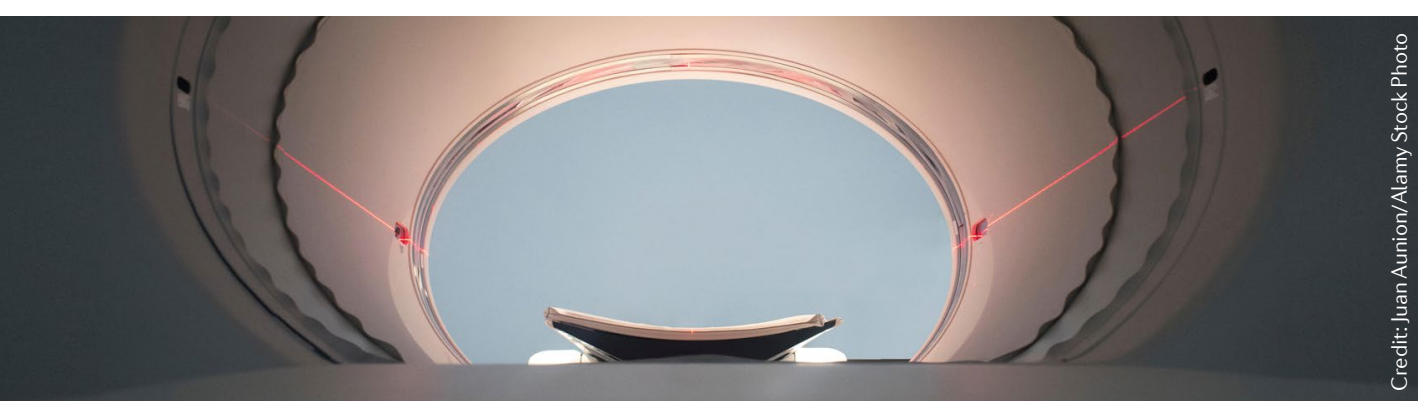

"PSMA PET-CT had greater impact on the way patients' disease was managed: $28 \%$ of men undergoing PSMA PET-CT had their treatment plans changed after the scans compared with $15 \%$ after conventional imaging," adds Hofman. Specifically, for first-line PSMA PET-CT, management of 20 of 148 (14\%) men was changed from curative to palliative therapy, and 22 men (14\%) underwent a different radiotherapy (7\%) or surgical technique (7\%). In 291 men who crossed over to second-line imaging, conventional imaging had a high or medium effect on management in $5 \%$ of men versus $27 \%$ of men for PSMA PET-CT. Overall, the final treatment decision was surgery in $42 \%$, radiotherapy in $41 \%$, androgen deprivation therapy alone in $9 \%$ and androgen deprivation therapy plus chemotherapy in $6 \%$ of men. Radiation exposure from first-line conventional imaging was $10.9 \mathrm{mSv}$ higher than that from PSMA PET-CT.

"On the basis of these results, we anticipate that guidelines will be modified with a view to recommending PSMA PET-CT, if available," concludes Hofman. "We are currently performing a health economic analysis to determine whether implementation of PSMA PET-CT is cost effective. If this new test is both more accurate, has higher utility and is cost effective, we expect PSMA PET-CT to become the new standard-of-care for initial imaging of men with prostate cancer and high-risk features before surgery or radiotherapy."

Clemens Thoma

ORIGINAL ARTICLE Hofman, M. S. et al. Prostate-specific membrane antigen PET-CT in patients with high-risk prostate cancer before curative-intent surgery or radiotherapy (proPSMA): a prospective, randomised, multicentre study. Lancet 395, 1208-1216 (2020) 